\title{
Antihypertensive treatment guided by genetics: PEARL-HT, the randomized proof-of-concept trial comparing rostafuroxin with losartan
}

\author{
Lorena Citterio ${ }^{1,2} \cdot$ Giuseppe Bianchi $\mathbb{1}^{2}$ - Giuseppe A. Scioli ${ }^{3}$ Nicola Glorioso ${ }^{4}$ Roberto Bigazzi ${ }^{5}$ Daniele Cusi ${ }^{6,7}$.

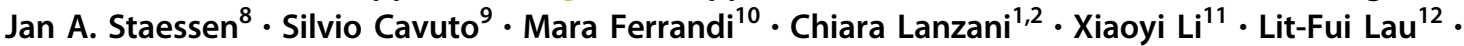 \\ Chern-En Chiang $^{13} \cdot$ Tzung-Dau Wang $^{14} \cdot$ Kang-Ling Wang $^{13} \cdot$ Patrizia Ferrari ${ }^{10} \cdot$ Paolo Manunta $\mathbb{D}^{1,2}$
}

Received: 29 June 2020 / Revised: 14 January 2021 / Accepted: 27 January 2021 / Published online: 1 March 2021

(c) The Author(s) 2021. This article is published with open access

\begin{abstract}
We compared a standard antihypertensive losartan treatment with a pharmacogenomics-guided rostafuroxin treatment in nevertreated Caucasian and Chinese patients with primary hypertension. Rostafuroxin is a digitoxigenin derivative that selectively disrupts the binding to the cSrc-SH2 domain of mutant $\alpha$-adducin and of the ouabain-activated Na-K pump at $10^{-11} \mathrm{M}$. Of 902 patients screened, 172 were enrolled in Italy and 107 in Taiwan. After stratification for country and genetic background, patients were randomized to rostafuroxin or losartan, being the difference in the fall in office systolic blood pressure (OSBP) after 2-month treatment the primary endpoint. Three pharmacogenomic profiles $(\mathrm{P})$ were examined, considering: P1, adding to the gene variants included in the subsequent $\mathrm{P} 2$, the variants detected by post-hoc analysis of a previous trial; $\mathrm{P} 2$, variants of genes encoding enzymes for endogenous ouabain (EO) synthesis ( $L S S$ and HSD3B1), EO transport (MDR1/ABCB1), adducin ( $A D D 1$ and $A D D 3$ ); $\mathrm{P} 3$, variants of the $L S S$ gene only. In Caucasians, the group differences (rostafuroxin $50 \mu \mathrm{g}$ minus losartan $50 \mathrm{mg}$ in OSBP $\mathrm{mmHg}$ ) were significant both in P2 adjusted for genetic heterogeneity (P2a) and P3 LSS rs2254524 AA [9.8 (0.6-19.0), $P=0.038$ and 13.4 (25.4-2.5), $P=0.031$, respectively]. In human H295R cells transfected with $L S S$ A and LSS C variants, the EO production was greater in the former $(P=0.038)$; this difference was abolished by rostafuroxin at $10^{-11} \mathrm{M}$. Chinese patients had a similar drop in OSBP to Caucasians with losartan but no change in OSBP with rostafuroxin. These results show that genetics may guide drug treatment for primary hypertension in Caucasians.
\end{abstract}

Supplementary information The online version contains supplementary material available at https://doi.org/10.1038/s41397021-00214-y.

Giuseppe Bianchi

bianchi.giuseppe@hsr.it

1 Genomics of Renal Diseases and Hypertension Unit, IRCCS San Raffaele Scientific Institute, Università Vita Salute San Raffaele, Milano, Italy

2 Università Vita Salute San Raffaele, Milan, Italy

3 Hypertension and Cardiovascular Prevention Center, Ospedale Ferdinando Veneziale, Isernia, Italy

4 Hypertension and Related Diseases Center, Department of Clinical and Experimental Medicine, University of Sassari, Sassari, Italy

5 Nephrology and Dialysis Unit, Livorno, Italy

6 Institute of Biomedical Technologies Milano National Research Council of Italy (CNR), Segrate, Milano, Italy

7 Bio4Dreams Scientific Unit, Bio4Dreams-Business Nursery for Life Sciences, Milano, Italy
8 Research Unit Hypertension and Cardiovascular Epidemiology KU Leuven Department of Cardiovascular Sciences, University of Leuven, Leuven, Belgium

9 Clinical Trials and Statistics Unit, Azienda USL-IRCCS di Reggio Emilia, Reggio Emilia, Italy

10 Windtree Therapeutics, Warrington, PA, USA

11 CVie Therapeutics, Taipei, Taiwan

12 Zhaoke (Guangzhou) Ophthalmology Pharmaceutical Limited, Guangzhou, China

13 General Clinical Research Center, Taipei Veterans General Hospital and National Yang-Ming University, Taipei, Taiwan

14 Cardiovascular Center and Division of Cardiology, Department of Internal Medicine, National Taiwan University Hospital and College of Medicine, National Taiwan University, Taipei, Taiwan 


\section{Introduction}

Rostafuroxin has been developed as a selective inhibitor of the ouabain blood pressure effects initially [1-3]. Later rostafuroxin has also been found to inhibit the effects of mutant adducin $[4,5]$ that was previously shown to be associated with hypertension in the Milan Hypertensive Rat Strain [6, 7] and in humans [8-11]. Rostafuroxin exerts its dual mechanism by selectively disrupting the binding to the cSrc-SH2 domain of mutant $\alpha$-adducin and of the ouabain-activated Na-K pump at $10^{-11} \mathrm{M}$, being inactive on the effects of wild adducin or of other 35 proteins or receptors involved in blood pressure regulation up to $10^{-5} \mathrm{M}[12,13]$. Therefore, rostafuroxin may be used as a safe, small molecule molecular probe [14, 15] to disentangle the genetic complexity of primary hypertension in humans and rats, facilitating and strengthening the comparisons between the two species. Rostafuroxin may display its therapeutic benefits by reducing the magnitude of cSrc triggered signal transduction, which favors renal tubular sodium reabsorption [9,10,15-17], hypertension, and cardiac/renal damage $[2,5,18-20]$ in carriers of gene variants affecting adducin function and/or ouabain tissue concentrations.

Four issues should be properly addressed when applying genetic tools to improve the beneficial effects of therapy of primary hypertension in clinical practice: (1) genetic heterogeneity, where different genes or different alleles within the same gene, may be involved in the regulation of the same phenotype [15, 21]; (2) epistasis, where many gene modifiers may either enhance or inhibit the effect of a given gene [22, 23]. Since the influence of modifiers differ among species, this hampers any kind of straightforward animal model-human comparison needed to assess causation; (3) moving from association to causation, relatively straightforward move in rodents with DNA manipulations [24], harder in humans, where a selective drug, that inhibits a specific genetically mediated pathway is needed; (4) avoiding the influence of the phases of hypertension and of the confounding effects of previous therapy that may take over, magnify or blunt the initial triggering mechanisms as shown in two well known causes of hypertension [25, 26]. After having obtained data supporting a plausible role of adducin variants or of endogenous ouabain (EO) in triggering hypertension in a rodent model and in humans $[1,2,6-11]$, we developed a strategy that takes into account the above mentioned four issues by, (1) applying the concept of genetic profile, in order to capture at least a portion of the genetic heterogeneity underlying adducin and EO functions [13]; (2) limiting the studies to newly discovered and never treated (naïve) patients [13]; (3) assessing causation with rostafuroxin $[4,12]$. By applying this strategy, we identified the genetic profile $2(\mathrm{P} 2)$, associated with the rostafuroxin blood pressure response, within the phase $2 \mathrm{a}$ OASIS-HT trial (ouabain and adducin for specific intervention on sodium in hypertension trial) [13]. P2 consists of variants at $\alpha$-adducin (ADD1 rs4961), $\gamma$-adducin (ADD3 rs3731566), lanosterol synthase (LSS rs2254524), $3 \beta$-hydroxysteroid dehydrogenase/ $\delta(5)-\delta(4)$ isomerase type 1 (HSD3B1 rs10923835), and ATP-binding cassette subfamily B member 1 (ABCB1/MDR1 rs1045642) genes, the last three being involved in the regulation of $\mathrm{EO}$ tissue levels.

We undertook the present phase $2 \mathrm{~b}$, double-blind, randomized, controlled PEARL-HT trial (PharmacogEnetic Assessment of Rostafuroxin vs Losartan in HyperTension) for validating the previous findings [13]. To this aim, we compared the office systolic blood pressure (OSBP) response to rostafuroxin and losartan in three subsets of naïve hypertensives, who differed for the gene variant number and the validity of previous existing data supporting their involvement. These three subsets included patients with at least (i) one combination of the above listed P2 gene variants, plus other variants that have been detected by a post-hoc analysis on the previous OASIS-HT trial, named Profile 1 (P1); (ii) one gene combination of the P2 listed above; (iii) homozygous wild CC (major) and homozygous for the mutant genotype AA (minor) of LSS rs2254524 (P3). To confirm the role of EO in our hypothesis, we transfected EO producing human adrenocortical cells (H295R) with LSS A or LSS C variants, to test if the previously shown increased EO production [13] in the former was inhibited by rostafuroxin at $10^{-11} \mathrm{M}$.

\section{Materials and methods}

\section{Study design, characteristics of the enrolled patients}

This PEARL-HT clinical trial (EudraCT, identifier 2010022073-34 and ClinicalTrials.gov, identifiers NCT01320397) phase $2 \mathrm{~b}$ multicenter, double-blind, double-dummy, fourarms, parallel group, and active comparator-controlled study was conducted in newly discovered and never-treated (naïve) hypertensive patients enrolled in 13 Italian and in 15 Taiwanese centers (Table S1). Besides the fact that we aimed to compare a generic drug as losartan that is one of the mainly used in the treatment for hypertension, the rationale underlying the choice of naïve patients and the losartan as a comparator is explained in the onlineonly Supplementary Data.

Two oral daily doses of rostafuroxin $(50$ and $500 \mu \mathrm{g})$ have been studied vs $50 \mathrm{mg}$ losartan both in Caucasian and Chinese patients (Fig. 1), who received both one capsule containing one of the two doses of rostafuroxin or 


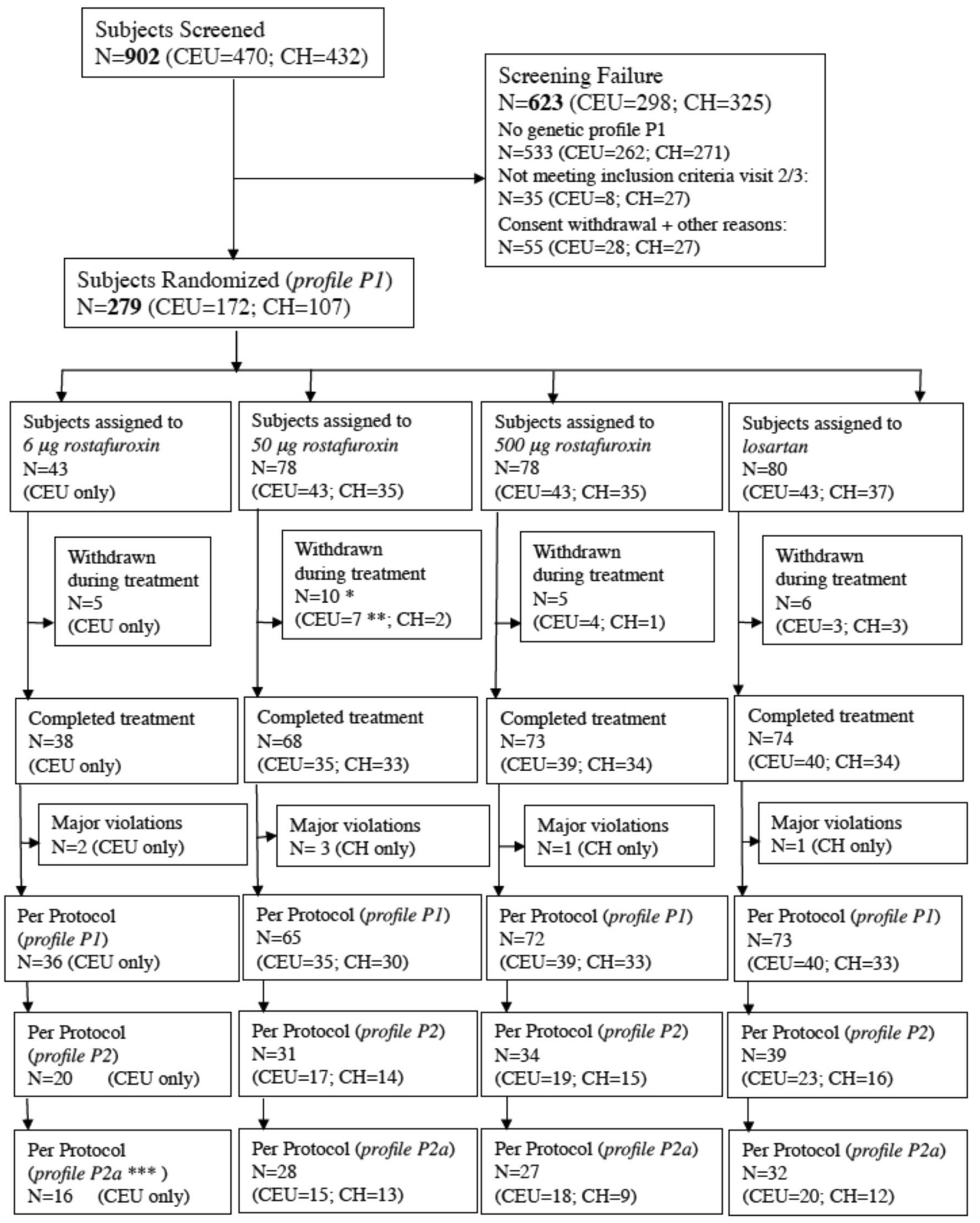

Fig. 1 CONSORT patients flow for PEARL-HT clinical trial. $* 1$ patient withdrawn without any treatment. **in the $50 \mu \mathrm{g}$ treatment group, the DO of four patients was requested by the sponsor because of a non-compliance problem in the initial rostafuroxin $50 \mu$ g capsules. ***to comply with the pre-specified definition of the PP population to evaluate the primary end point, the patients randomized as the carrier

placebo of rostafuroxin and one tablet containing $50 \mathrm{mg}$ losartan or placebo of losartan. The treatment period lasted 9 weeks. An additional arm of $6 \mu \mathrm{g}$ rostafuroxin was carried of a gene pair alone or in combination, which are not present in all the four arms, have to be removed from the profile $\mathrm{P} 2$ to obtain the profile P2a. Therefore, four, two, one, and three patients were removed from the 6,50 , and $500 \mu \mathrm{g}$ rostafuroxin and losartan arms, respectively. CONSORT, Consolidated Standards of Reporting Trials.

out in Caucasian patients only. The chemical and pharmacologic characteristics of rostafuroxin are described elsewhere $[4,5]$. 
Table 1 Overall profile 1 (P1) in Caucasian and Chinese.

\begin{tabular}{|c|c|}
\hline Caucasian & Chinese \\
\hline$A D D 1$ rs4961 GT + TT \& $A D D 3$ rs3731566 GG & $A D D 1 \mathrm{rs} 4961 \mathrm{GT}+\mathrm{TT} \& \underline{A D D 3 r s 2501574 \mathrm{TT}}$ \\
\hline $\begin{array}{l}A B C B 1 / M D R 1 \text { rs1045642 TT \& HSD3B1 } \\
\text { rs10923835 AT }+ \text { TT }\end{array}$ & $\begin{array}{l}A B C B 1 / M D R 1 \text { rs1045642 TT \& } \underline{H S D 3 B 1} \\
r=117585927 C G+C C\end{array}$ \\
\hline $\begin{array}{l}L S S \mathrm{rs} 2254524 \mathrm{CA}+\mathrm{AA} \& A B C B 1 / M D R 1 \\
\text { rs1045642 CC }\end{array}$ & $\begin{array}{l}L S S r s 2254524 \mathrm{CA}+\mathrm{AA} \& A B C B 1 / M D R 1 \\
\text { rs1045642 CC }\end{array}$ \\
\hline$L S S r s 2254524$ AA \& ADD1 rs4961 GT + TT & $L S S$ rs2254524 AA \& ADD1 rs4961 GT + TT \\
\hline $\mathrm{rs} 16893522=\mathrm{AA}$ & $\mathrm{rs} 16893522=\mathrm{AA}$ \\
\hline $\mathrm{rs} 2345088=\mathrm{TT}$ & $\mathrm{rs} 2345088=\mathrm{TT}$ \\
\hline $\mathrm{rs} 2461911=\mathrm{AA}$ & $\operatorname{rs} 2461911=\mathrm{AA}$ \\
\hline $\mathrm{rs} 16877182=\mathrm{CT}$ & rs $16877182=\mathrm{CT}$ \\
\hline $\mathrm{rs} 12513375=\mathrm{GG}$ & $\mathrm{rs} 12513375=\mathrm{GG}$ \\
\hline HSD3B1 rs947130 GG \& NEDD4L rs4245268 CC & HSD3B1 rs947130 GG \& NEDD4L rs4245268 CC \\
\hline $\begin{array}{l}A B C B 1 / M D R 1 \text { rs } 1045642 \mathrm{CC} \& A G T R 1 \\
\text { rs } 2131127 \mathrm{CC}\end{array}$ & $\begin{array}{l}A B C B 1 / M D R 1 \text { rs } 1045642 \mathrm{CC} \& A G T R 1 \\
\text { rs2131127 CC }\end{array}$ \\
\hline$A D D 2$ rs4984 CC \& rs10502933 CT & $\underline{A D D 2}$ rs12470211 $A G+G G \&$ rs $10502933 \mathrm{CT}$ \\
\hline$L S S$ rs2254524 AA \& WNK1 rs880054 AG + GG & $L S S$ rs 2254524 AA \& WNK1 rs880054 AG + GG \\
\hline
\end{tabular}

Genotype combinations in P1 and P2 (in bold) both in Caucasian and in Chinese. Genotype variations specific to Chinese are in italics and underlined, as explained in the online-only Supplementary Data. P1 was composed by adding to the gene variants included in the $\mathrm{P} 2$, the variants detected by post-hoc analysis of a previous OASIS-HT trial; P2, variants of genes encoding enzymes for endogenous ouabain (EO) synthesis (LSS and HSD3B1), EO transport (MDR1/ABCB1), adducin ( $A D D 1$ and $A D D 3$ ); P3, variants of the $L S S$ gene only (homozygous wild CC (major) and homozygous for the mutant genotype AA (minor)).
The eligibility of the naïve hypertensive patients included: age between 25 and 60 years, being carrier of one or a combination of polymorphisms of the P1 (Table 1 and Table S2), with at least $50 \%$ of the enrolled patients having a combination of gene variants of the original P2 (OASIS-HT) [13], to have already undertaken lifestyle recommendations and still having sitting OSBP ranging between 140 and $169 \mathrm{mmHg}$ and Office Diastolic BP (ODBP) ranging between 85 and $100 \mathrm{mmHg}$. Though eligible patients should not have been previously treated with any specific antihypertensive drug, a short period of treatment (no longer than seven days) was allowed before enrolment if a washout of about 1 month could be applied before randomization. Other criteria for non-eligibility were: statin treatment, presence of renal or adrenal secondary forms of hypertension, fasting plasma glucose $>125 \mathrm{mg} / \mathrm{dl}(6.9 \mathrm{mmol} / \mathrm{L})$, estimated creatinine clearance $\leq 50 \mathrm{ml} / \mathrm{min}$, and if women, being pregnant, nursing, or of childbearing potential not taking anti-contraceptive medication. Local ethics committees approved the research protocol (Text S1).

\section{Central genotyping and randomization}

Genomic DNA was extracted from peripheral blood samples collected at screening visit (visit 1) and genotyped using TaqMan OpenArray Genotyping System (Life Technologies, Foster City, CA) at San Raffaele Scientific Institute (Milan, Italy) according to the manufacturer's instructions. See more details in the online-only Supplementary Data.

Assignment to treatment groups was determined by a computer-generated random sequence using an interactive web-based response system as described in the onlineonly Supplementary Data.

\section{Selection of the eligible patients and study procedures}

The eligible patients were identified either by a Clinic Center Physician or a General Practitioner who prescribed life style changes. Then, these patients were followed by the physician in charge of this study at the hospital center, who carried out the following visits: at visit 1 screening: collection signed Informed Consent, check prior and concomitant medications, blood sampling for chemistry, pregnancy test, genotyping, ECG, providing recipient for 24-hour urine collection (to measure sodium excretion), OSBP, and ODBP. Check of verifiable inclusion/exclusion criteria (see Protocol in Text S1). Visit 1 was followed by other two run in visits 2 and 3, and post-randomization visits 4, 5, 6, and 7 at 2, 4, and 6 weeks, respectively. Visit 7 was carried out one day after visit 6 , just to remove the 24hour Ambulatory Blood Pressure (24h-ABPM) device. The baseline 24h-ABPM was carried out between visit 2 and visit 3 . At visit 3 final inclusion/exclusion criteria, randomization and dispensing the drug boxes for the subsequent drug period; at visits $3,4,5$, and 6 office $\mathrm{BP}$ and HR 
measurements as at visits 1 and 2, check of concomitant disease, medications, adverse events, and drug accountability; measurements, blood sampling for chemistry, and pregnancy test.

\section{Definition of confounders, distinction between primary and secondary variables and statistical analysis}

The rationale for choosing the genetic background and the basal BP as confounders with the distinction between primary and secondary variables is described in the onlineonly Supplementary Data.

The average of the last three readings of OSBP were considered in the statistical analysis. All BP variables were assumed to be normally distributed. Standing the definition of the primary objective in the protocol, as the OSBP difference between visit 6 and visit 3, the primary efficacy analyses were carried out in the Per Protocol (PP) population, while the safety analyses were carried-out in the safety population. The one-way analysis of variance (ANOVA) was tested for the first step of analysis, with the corresponding mean values with SD. The comparison between treatment groups has been carried out with an analysis of covariance (ANCOVA) model with change from baseline to visit 6 as dependent variable, treatment, country, and baseline as covariate and the data presented as mean with standard error (SE) and 95\% CI. The Levene's test confirmed the homoscedasticity of variance of OSBP between the groups of comparison.

\section{Outcomes}

The relatively long treatment duration of 2 months, implying the use of a PP population for primary analysis instead of an Intention to Treat (ITT) population, is based on the similarities between the rostafuroxin and spironolactone action mechanisms and pharmacodynamics. Both drugs counteract the excess renal tubular sodium reabsorption responsible for BP increase in carriers of the P2 or adrenal adenoma, respectively. Indeed, a treatment of several weeks is needed to fully exploit the spironolactone antihypertensive activity [27].

The two primary objectives were to demonstrate that at least one of the two highest doses of rostafuroxin (50 or $500 \mu \mathrm{g}$ ) reduces significantly OSBP at visit 6 compared to $50 \mathrm{mg}$ losartan, in patients bearing either:

(1) at least one pair of gene variants of the P2, or

(2) at least one variant or pair of variants included in the P1.

Because the considerable variation among the frequencies of the four gene-pairs of the P2 detected before unblinding, in the statistical analysis plan (SAP) (Text S2) and in the data review report (DRR) (Text S3) it was stated: "the need to minimize the genetic heterogeneity across the treatment arms by maximizing the frequency similarity of the gene-pairs across the different arms".

Thus, the results of the treatment efficacy within the four arms are given both as a $\mathrm{P} 2$ and $\mathrm{P} 2 \mathrm{a}$, representing, respectively, the unadjusted and the adjusted results for the genetic heterogeneity, being the latter considered the one to use to assess the primary end-point, as pre-specified in the SAP. An additional analysis, also pre-specified in SAP, has been performed considering LSS rs2254524 polymorphism (genotypes AA and $\mathrm{CC}$ ) alone to confirm previous data [13].

\section{LSS expression analysis and endogenous ouabain quantification in H295R cells}

Transfection with LSS major (C-642Val) and minor (A642Leu) variants was investigated in human adrenocortical cells (H295R cells), as described [13]. EO was determined by radioimmunoassay on $\mathrm{C} 18$-extracted samples using a specific antiserum [3]. More details of these methods are given in the online-only Supplementary Data.

\section{Study power and cell data statistical analysis}

According to the assumptions in the protocol (Text S1) and SAP (Text S2), a total sample size of 120 patients $(50 \%$ of the total sample), i.e., 40 patients in each group, is suitable for discriminating an OSBP mean difference of $6.5 \mathrm{mmHg}$ between the groups of the two highest doses of rostafuroxin and losartan, with a standard deviation of $10 \mathrm{mmHg}$, a power of $80 \%$, an alpha level of 0.05 , two tailed test, in the PP and ITT genetic P2 populations, and assuming a $10 \%$ drop-out rate. However, as shown in Table 2 discussed in "Results", the lack of OSBP response to rostafuroxin in Chinese patients required an analysis by country, thus the above sample size was reduced by $50 \%$.

For H295R cell culture transfection, data were expressed as means \pm SE with $95 \%$ CI. Statistical comparisons were analyzed by Student's t test or one-way ANOVA. When the individual measurements were below 5 , the single values and means were shown. A $P$ value of 0.05 was chosen for statistical significance.

\section{Results}

Globally, 902 hypertensives were screened (Fig. 1). Of them, 470 were screened and 172 randomized in Italy between June 26, 2013 and January 8, 2016, while 432 were screened and 107 randomized in Taiwan between December 18, 2015 and December 6, 2017. Only patients carrying at least one or a pair of gene variants included in the genetic profile P1 were randomized (Table 1), with at least $50 \%$ of them having the 
Antihypertensive treatment guided by genetics: PEARL-HT, the randomized proof-of-concept trial...

351

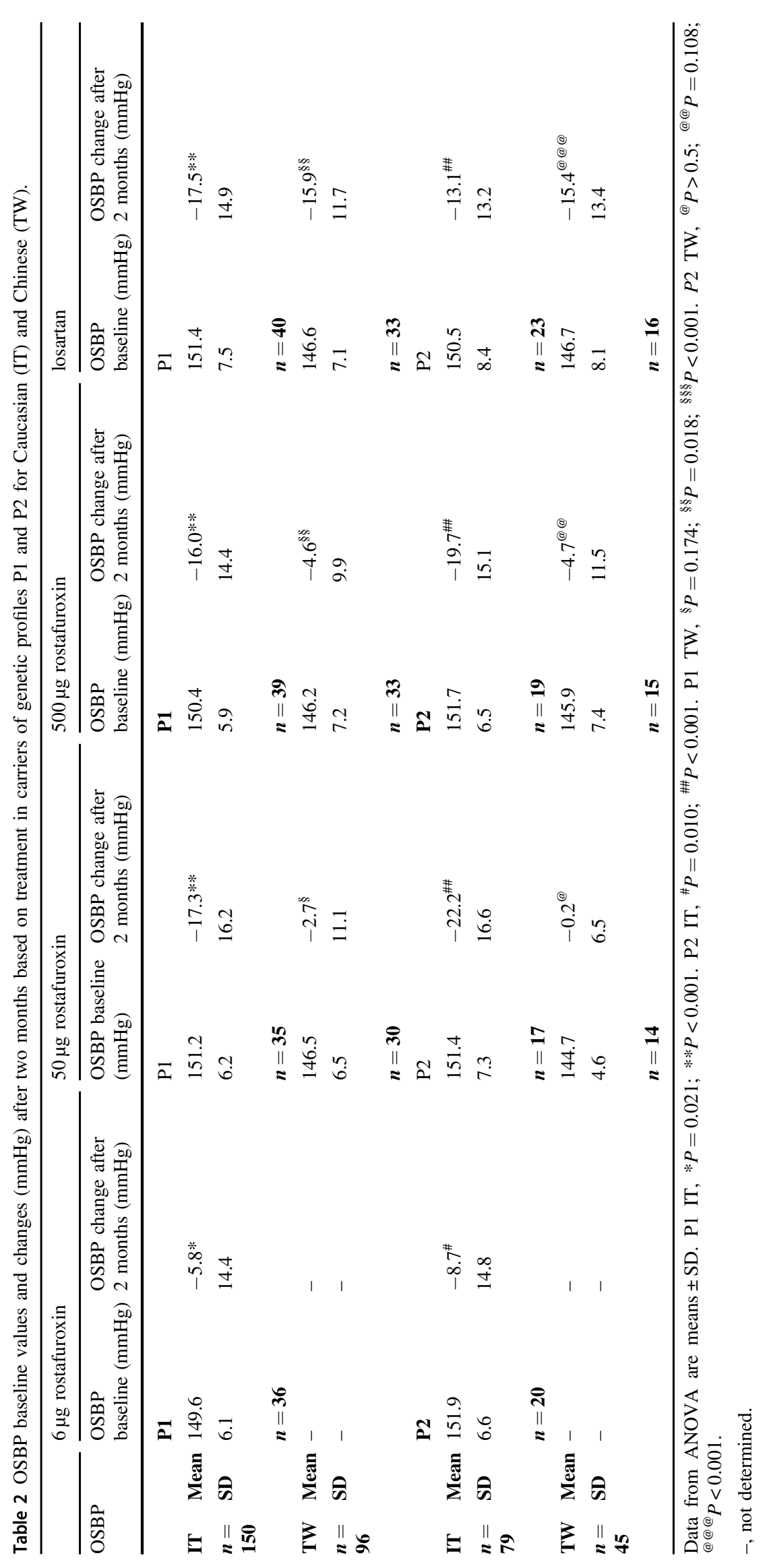

SPRINGER NATURE 
combination of the gene variants of the original P2 (OASISHT) [13]. The clinical characteristics of the Caucasians are given in Table S3, while those of Chinese are in Table S4. The percentage of male was about twice that of female in both populations $(70 \%$ vs $30 \%$ in Caucasians, $64 \%$ vs $36 \%$ in Chinese). No mean age difference was shown between genders in both the Caucasians and the Chinese $(47.8( \pm 7.4)$ males vs $50.8( \pm 5.9)$ females, and $45.9( \pm 8.6)$ males vs 48.9 $( \pm 6.4)$ females, respectively). As explained in "Outcome" and shown in the flowchart (Fig. 1), the P2 and P2a represented the unadjusted and the adjusted results for the genetic heterogeneity among study arms, respectively, being the latter considered the one to be used to assess the primary end point, as pre-specified in the SAP (Text S2).

\section{Chinese patients do not respond to rostafuroxin but to losartan}

OSBP did not change in Chinese carrying either the P1 or $\mathrm{P} 2$ at any tested dose of rostafuroxin (Table 2). The lack of response in Chinese $(-0.2 \pm 6.5 \mathrm{mmHg}$ with $50 \mu \mathrm{g}$ rostafuroxin) implies an inhibition of the "placebo" response to rostafuroxin treatment, as previously observed in Caucasians carrying the P2 [13]. Conversely, the efficacy of losartan was similar in carriers of the P2 of both ethnic groups. Therefore, only the data on Caucasian are presented and discussed hereafter.

\section{Caucasian carriers of the P2 and P2a, but not of the $P 1$, have a greater fall of OSBP to rostafuroxin than to losartan}

In Caucasian carrying the P1, both all rostafuroxin doses and losartan produced a statistically significant OSBP fall $(P=$ 0.021 for rostafuroxin $6 \mu \mathrm{g}, P<0.001$ for the other rostafuroxin doses and losartan; Table 2, ANOVA test). The fall of OSBP to losartan and to each of the highest doses of rostafuroxin were very similar $(P>0.5$; ANOVA test), (Table 2$)$. In P2a Caucasians, the OSBP decrease was statistically significant at any tested dose of rostafuroxin (Table 3 and Table S5) being greater with $50 \mu \mathrm{g}$ rostafuroxin than with $500 \mu \mathrm{g}$ rostafuroxin, even though this difference did not reach statistical significance (Table 3 and Table S5). This trend towards a bell-shaped curve of the rostafuroxin dose-effect relationship, with the peak at $50 \mu \mathrm{g}$, is consistent with previously published preclinical and clinical data of the OASISHT study [13]. Compared to losartan, the OSBP change was greater with the two highest doses of rostafuroxin, being this difference larger and statistically significant with the dose of $50 \mu \mathrm{g}$ : $-9.8 \mathrm{mmHg}(-19.0 ;-0.6, \quad[95 \% \mathrm{CI}]) \quad P=0.038$ (Table 3 and Table S5). Fig. 2a shows the OSBP values, in carriers of the $\mathrm{P} 2 \mathrm{a}$, at the different time points. The OSBP changes between visit 6 (at 9 weeks of treatment) and visit 5 (at 5 weeks of treatment) was $+2.6 \pm 1.7 \mathrm{mmHg}$ and $-4.2 \pm$ $4.3 \mathrm{mmHg}($ mean $\pm \mathrm{SE})(P=0.113)$ for losartan and rostafuroxin at $50 \mu \mathrm{g}$, respectively.

Table 3 also shows the OSBP changes in P2 carries, not adjusted for the genetic heterogeneity. The magnitude of the difference between rostafuroxin and losartan was slightly lower than that of $\mathrm{P} 2 \mathrm{a}(-22.2 \mathrm{vs}-23 \mathrm{mmHg})$, as the significance $(P=0.062$ vs $P=0.038)$.

\section{The LSS rs2254524 AA and CC genotypes differently affect the pressor response to rostafuroxin and to losartan}

To confirm previous findings [13], a pre-specified analysis was carried out on the pressor response to rostafuroxin and losartan in the context of LSS rs2254524 AA and CC genotypes alone and summarized in Fig. 2b. Compared to losartan, $50 \mu \mathrm{g}$ rostafuroxin produced a larger OSBP fall in LSS AA (mean mmHg, $(95 \% \mathrm{CI}))-13.4(-25.4 ;-1.5)$ $(P=0.031)$ but a smaller one in LSS CC $+7.3(-3.9$; $+18.6)$. The interaction between genotypes and drug responses was statistically significant $(P=0.023$ for OSBP and $P=0.013$ for ODBP). As in the P2a carriers, also in the LSS AA carriers, the OSBP responses to the three doses of rostafuroxin displayed a trend towards a bell shape curve, with the peak effect at the dose of $50 \mu \mathrm{g}$ (Fig. 3a).

Fig. 4 pools OSBP changes from Tables 2, 3 and Fig. 2b allowing a comparison among the various subsets of patients, according to their genotypes. The differential OSBP lowering effect of losartan and rostafuroxin was dependent upon the underlying genotypes.

Thirteen patients dropped out at visit 4 or before and six at visit 5. As shown in Table S6, one subject treated with $500 \mu \mathrm{g}$ rostafuroxin reported a skin rash as adverse event. Detailed safety data for treatment-emergent adverse drug reactions in Caucasians and Chinese are reported in Table S7. No serious adverse events were reported in Caucasian, as described in Table S8, while one was present in Chinese and regarded an episode of severe vertigo.

\section{The OSBP changes to $50 \mu \mathrm{g}$ rostafuroxin in LSS rs2254524 AA and CC carriers are similar to that of previous OASIS-HT trial}

The previous OASIS-HT study aimed at assessing the hypotensive efficacy of rostafuroxin on individuals bearing specific allele combinations of the P2. Compared to PEARL-HT, it had lower duration of treatment (5 weeks) and placebo was used as a head-to-head comparator [13]. Furthermore, due to a low number of patients, the results of the rostafuroxin effect were provided by grouping the lower and the higher doses. To verify whether the preliminary results of the OASIS-HT trial could be confirmed in the 


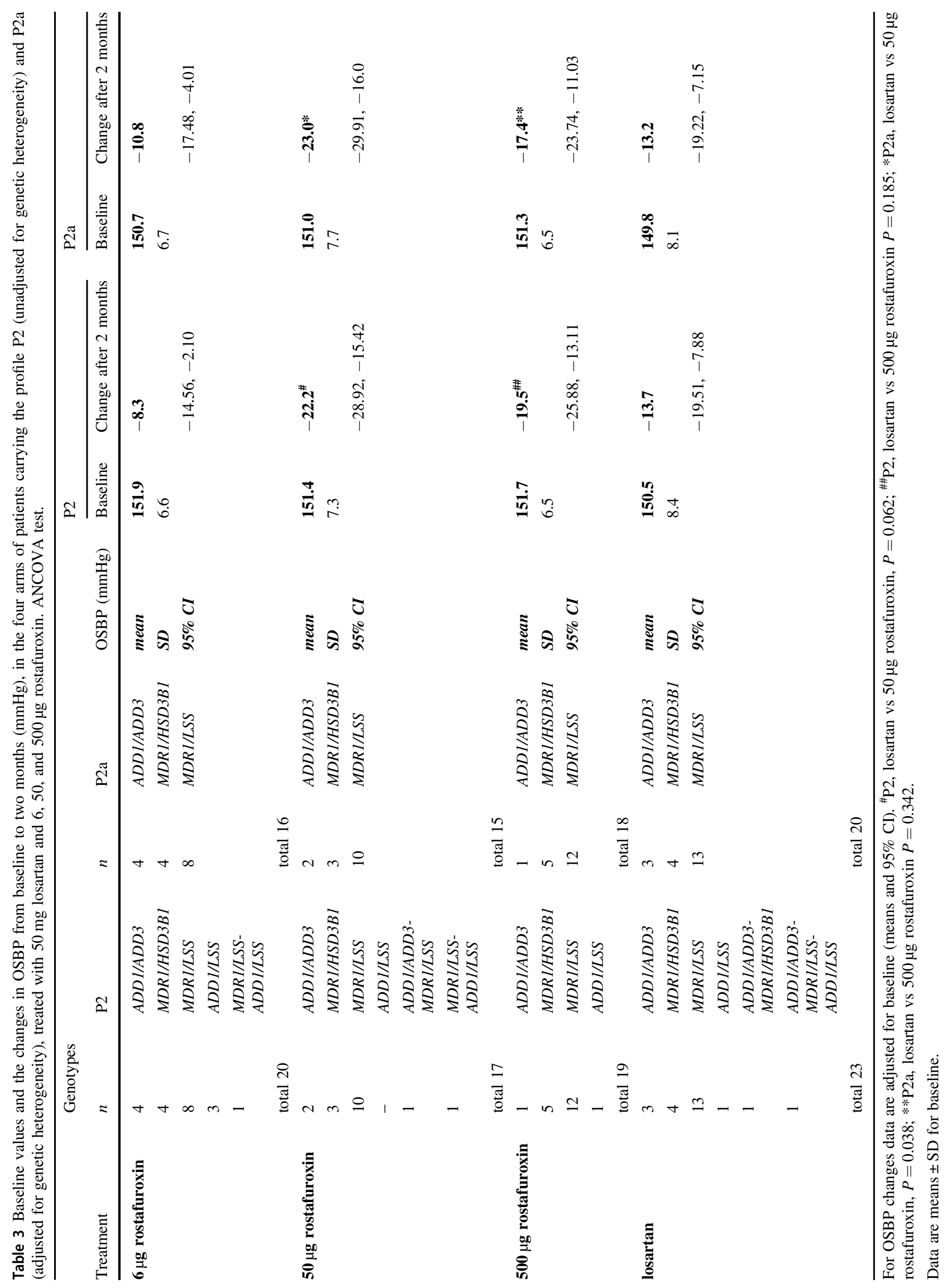


Fig. 2 Blood pressure changes after rostafuroxin or losartan treatment in carriers of the profile P2a or of $L S S$ AA or CC genotypes. A Time-course of mean OSBP changes ( $\mathrm{mmHg}$ ) at visit 1 , at baseline, and visits 4 ( 2 weeks), 5 (4-5 weeks), and 6 ( 9 weeks) in profile $\mathrm{P} 2 \mathrm{a}$ in the four treatments groups. Data are means \pm SE. B OSBP and ODBP fall ( $\mathrm{mmHg}$ ) in LSS AA and $\mathrm{CC}$ carriers treated with $50 \mathrm{mg}$ losartan or $50 \mu \mathrm{g}$ rostafuroxin. ANCOVA test. Data adjusted for baseline are means $( \pm$ SE). $\Delta=$ delta OSBP or ODBP. Numbers in the columns refer to each subgroup sample size. $* \Delta=13.4 \mathrm{mmHg}$ OSBP for $L S S$ AA, $50 \mathrm{mg}$ losartan vs $50 \mu$ g rostafuroxin, $P=0.031$; ** LSS x treatment (OSBP), $P=0.023$; *** $\Delta=9.1 \mathrm{mmHg}$ ODBP for $L S S$ AA, $50 \mathrm{mg}$ losartan vs $50 \mu \mathrm{g}$ rostafuroxin, $P=0.095 ; * * * * L S S \times$ treatment (ODBP), $P=0.013$. C OSBP fall (mmHg) in LSS AA and CC individual carriers treated with $50 \mu \mathrm{g}$ rostafuroxin, in PEARLHT and OASIS-HT trials. The values below the histograms are the OSBP levels reached by the individual patients at the end of the treatment period.

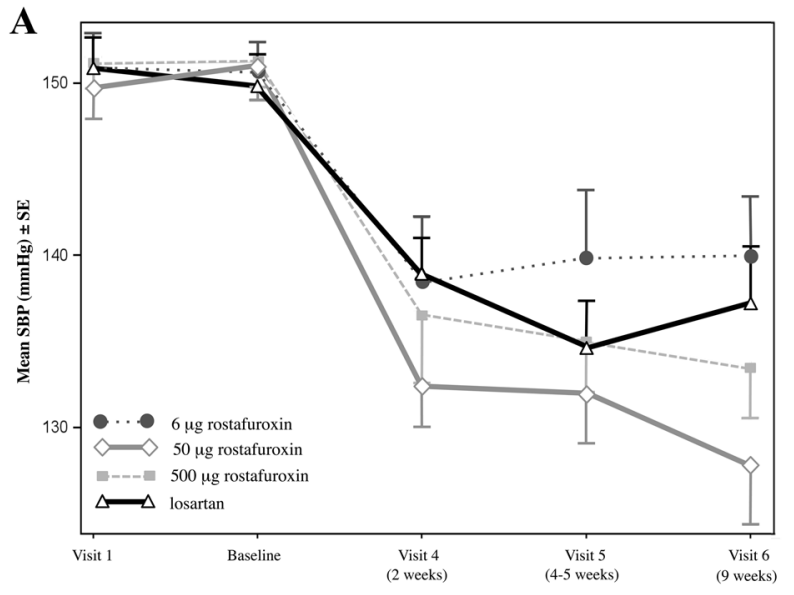

B

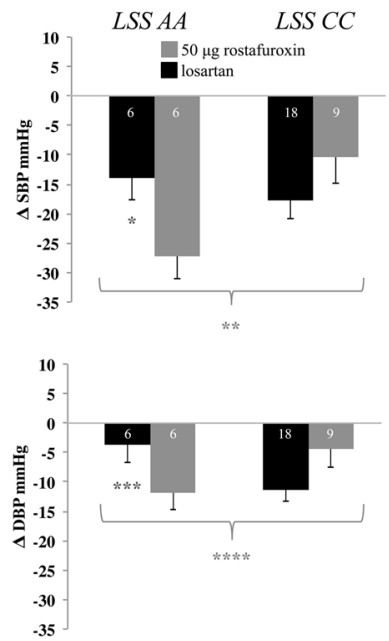

C

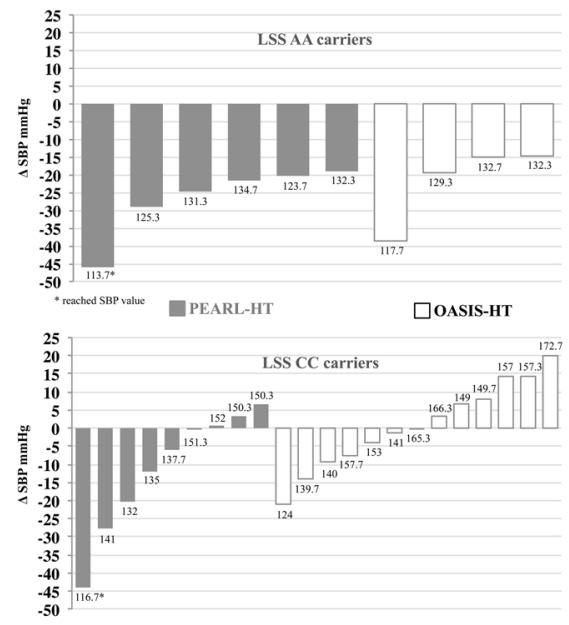

present study, the individual OSBP data of the OASIS-HT patients treated with $50 \mu \mathrm{g}$ rostafuroxin and carrying the P2 (6 patients) or the LSS AA (4 patients) and LSS CC (13 patients) genotypes were retrieved. These baseline values and changes after 5 weeks of treatment are reported in Table S9, where also the patients treated with losartan are included. Treatment with $50 \mu \mathrm{g}$ rostafuroxin reached the OSBP target level of $135 \mathrm{mmHg}$ in all 10 patients carrying the LSS AA genotype, but only in 4 out of 22 patients carrying the LSS CC genotype (Fig. 2c). It is noteworthy that a change of only one aminoacid, from Valine to Leucine at position 642 in the $L S S$ protein, produced such a remarkable difference in the OSBP response to rostafuroxin. In Table S10 are shown the pooled OSBP responses to rostafuroxin $50 \mu \mathrm{g}$ and to losartan $50 \mathrm{mg}$ from the previous clinical trials [13] and the present PEARL-HT studies in the $\mathrm{P} 2, L S S$ AA, and LSS CC Caucasian carriers. The level of statistical significance of the difference between rostafuroxin and losartan OSBP responses found in the present PEARL-HT study is increased in the pooled data. In particular, in the P2 carriers, unadjusted for genetic heterogeneity, the $P$ values were 0.062 and 0.035 in the PEARL-HT and pooled data, respectively.

For the secondary end-points, ODBP, night- and 24hABPM, a statistically significant fall of BP was observed in the losartan and rostafuroxin $50 \mu \mathrm{g}$ treated patients, but these BP changes were not different between the two treated groups, as reported in the online-only Supplementary Data.

\section{Rostafuroxin differently affects LSS mRNA level and EO production in human adrenocortical cells transfected with LSS rs2254524 variants}

We previously showed that, compared to the cells transfected with the LSS major allele (C-642Val), the cells transfected with the minor allele (A-642Leu) have lower LSS mRNA levels and higher EO in the supernatant [13]. Here, we confirm these previous data. Compared to the major $L S S$ C transfected cells values at baseline, the minor $L S S$ A displayed lower levels of $L S S$ mRNA (arbitrary units from $295 \pm 49$ to $149 \pm 0.42 ; P=0.003$ ), associated with 
Fig. 3 Rostafuroxin effects in Caucasian carriers of LSS AA and CC genotypes on OSBP changes and in $H 295 R$ cells transfected with LSS A or C variants on LSS mRNA and EO levels. A OSBP fall (mmHg) in LSS AA and CC carriers treated with $50 \mathrm{mg}$ losartan or 6,50 , and $500 \mu \mathrm{g}$ rostafuroxin. Data adjusted for baseline are means $\pm \mathrm{SE}$. * ANCOVA (mean, 95\% CI) $50 \mu \mathrm{g}$ rostafuroxin $L S S$ AA $-25.2(-34.3 ;-16.0)$ vs CC $-12.2(-19.6 ;-4.7), P=$ 0.034 . B $L S S$ mRNA levels in H295R transfected cells at baseline and incubated with different concentrations of rostafuroxin. Single data of each subgroup are represented by a dot. \# $T$ test at baseline (mean, 95\% CI) major C 295.7 (222.6; 368.7) vs minor A 149.8 (87.3; 212.3), $P=0.003$. \#\# $T$ test in the presence of rostafuroxin $10^{-11} \mathrm{M}$, (mean, $95 \% \mathrm{CI}$ ) major C $141.3(45.2 ; 237.5)$ vs minor A $442.0(271.2 ; 612.8), P=$ 0.0028. C EO levels in H295R transfected cells incubated with $10^{-11} \mathrm{M}$ rostafuroxin. Data are means \pm SE. $\S T$ test at baseline (mean, 95\% CI) major C 5.5 $(3.6 ; 7.4)$ vs minor A 9.2 (6.0; $12.5), P=0.038$. $\S \S T$ test in the presence of rostafuroxin $10^{-11} \mathrm{M}$ (mean, 95\% CI) major C 5.2 $(2.8 ; 7.5)$ vs minor A $6.6(5.0$; 8.2), $P=0.26$

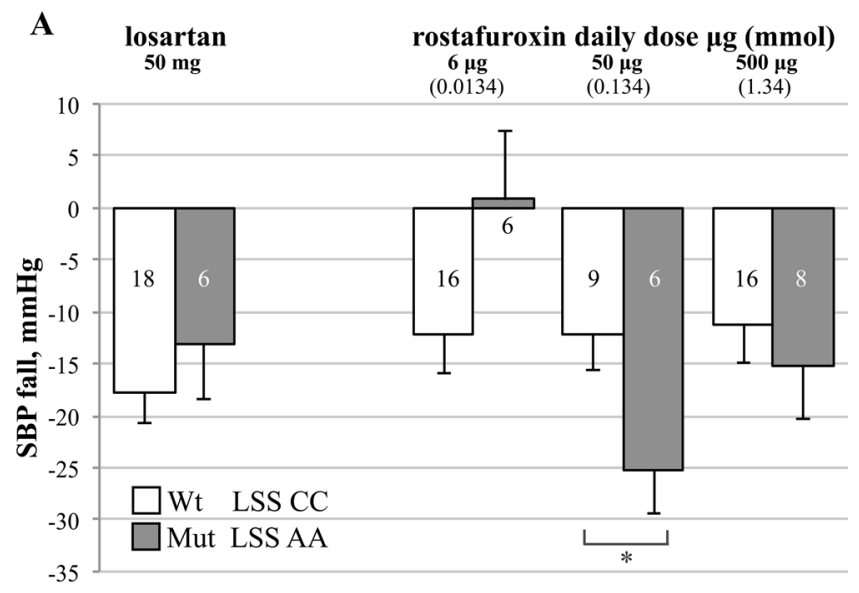

B

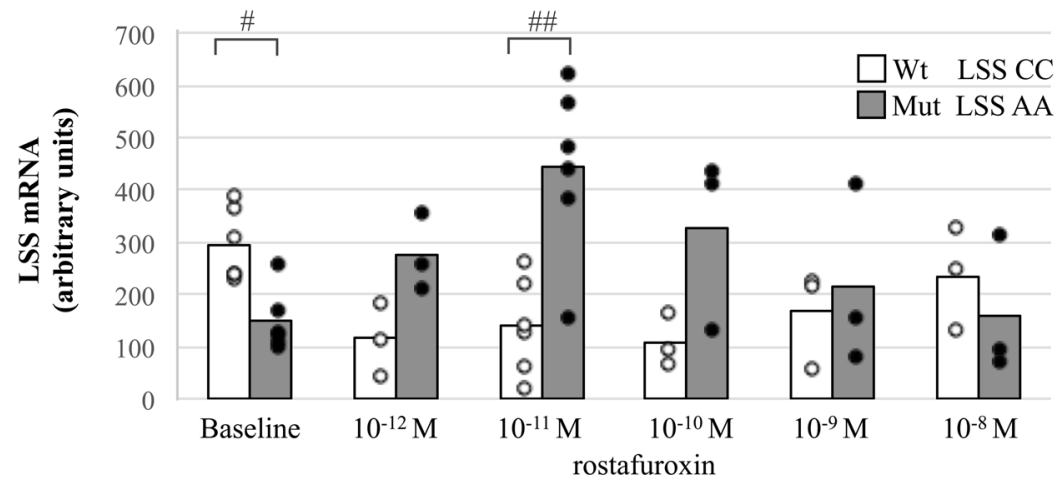

C

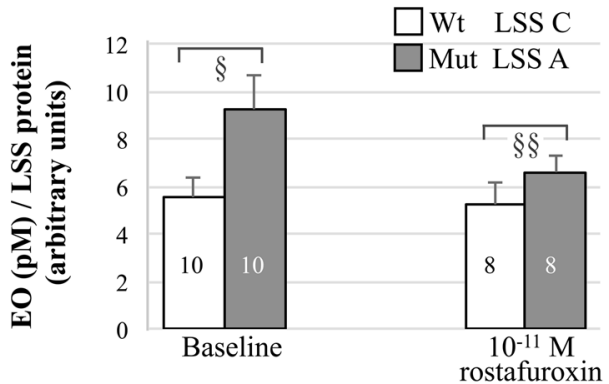

higher EO values $(\mathrm{EO}(\mathrm{Pm})$ per unit of $L S S$ protein from $5.5 \pm 0.8$ to $9.2 \pm 1.4 ; P=0.038$ ), as shown in Fig. $3 \mathrm{~b}$, c, respectively.

Incubation with rostafuroxin at increasing concentrations from $10^{-12}$ to $10^{-8} \mathrm{M}$ for $24 \mathrm{~h}$ differentially affected mRNA concentrations in cells transfected with the major or the minor LSS alleles. In minor A background, the LSS mRNA increased according to a bell shape curve with a peak at $10^{-11} \mathrm{M}$ rostafuroxin, while in the major $\mathrm{C}$, at the same rostafuroxin concentration, mRNA level decreased (Fig. 3b). Furthermore, the baseline difference of EO levels observed between major $\mathrm{C}$ and minor A transfected cells (Fig. 3c) was abolished by incubation with rostafuroxin at $10^{-11} \mathrm{M}$.

\section{Discussion}

The main findings of the present study were as follows: (1) Caucasians naïve patients carrying the genetic profile $\mathrm{P} 2 \mathrm{a}$ or LSS AA genotype had larger OSBP fall to rostafuroxin $50 \mu \mathrm{g}$ than to losartan; (2) rostafuroxin, in the H295R cells transfected with LSS A or LSS C allele, abolished the baseline increased EO production in the LSS A cells over that of LSS C cells, and enhanced the depressed levels of LSS mRNA in the former cells, thus demonstrating a causal link between genotypes and rostafuroxin's effect also at cellular levels.

The efficacy of rostafuroxin in the P2a and LSS AA Caucasians carriers was similar to that observed in 
Fig. 4 Summary of OSBP fall $(\mathrm{mmHg})$ in $\mathbf{5 0} \mu \mathrm{g}$ rostafuroxin and in losartan treatment arms across the different genetic backgrounds, Caucasian subgroups.

Numbers in the columns refer to each subgroup sample size. ANCOVA test. Data adjusted for basal OSBP are means \pm SE $* \Delta=9.8 \mathrm{mmHg}$ OSBP for P2a, $50 \mathrm{mg}$ losartan vs $50 \mu \mathrm{g}$ rostafuroxin, $P=0.038$; ** $\Delta=$ $13.4 \mathrm{mmHg}$ OSBP for LSS AA, $50 \mathrm{mg}$ losartan vs $50 \mu \mathrm{g}$ rostafuroxin, $P=0.031$.

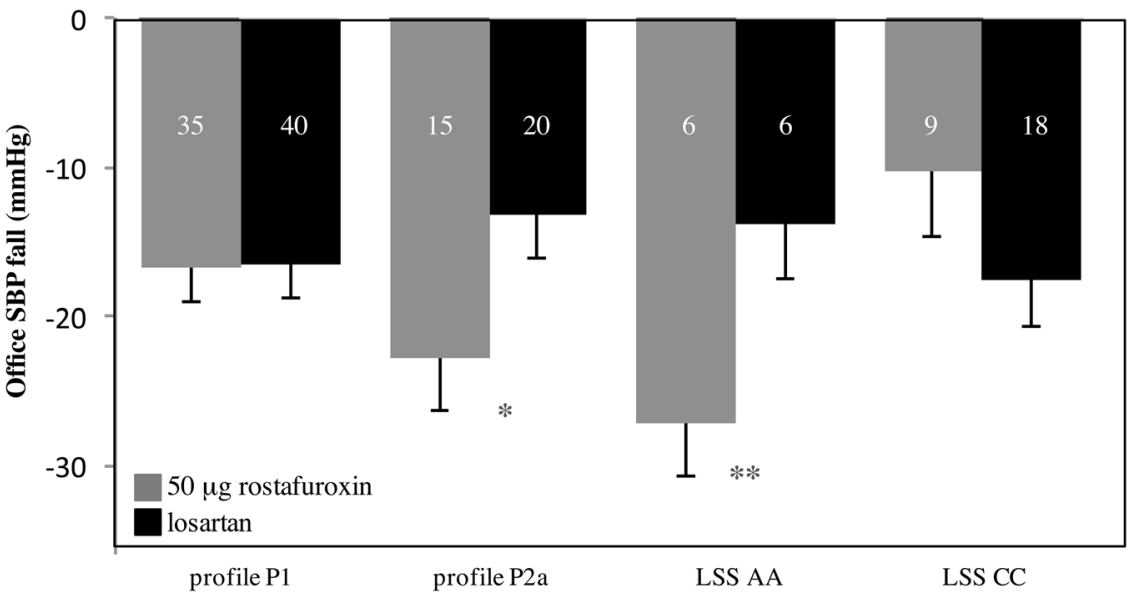

the previous OASIS-HT trial [13]; therefore, the present results may be considered a replication of previously published results [13]. Moreover, the OSBP differences between rostafuroxin and losartan were larger than those described in previous studies where a head-to-head comparison among antihypertensive drugs was tested $[28,29]$ and, most importantly, they were genotype dependent. These OSBP changes were not associated with any serious adverse event, while the adverse events were not different from those of the comparator that was placebo in the OASIS-HT e and losartan in the present trial.

The dose-effect relationship of rostafuroxin tended to have a bell-shaped trend, particularly in carriers of LSS AA, having a peak effect at $50 \mu \mathrm{g}$. A similar trend towards a bellshape curve was observed in the previous published OASIS-HT study [13] and is consistent with the previous and present data obtained in cell-free system and isolated rat and human transfected cells $[2,5,12,13]$. In those settings, rostafuroxin selectively inhibits the altered biochemical step common to both mutant adducin and ouabain pressor mechanisms at $10^{-10}$ or $10^{-11} \mathrm{M}$, being this effect lower both at higher and lower concentrations. Moreover, there is a plausible correspondence between the very small daily oral dose of $50 \mu \mathrm{g}$ (equal to 0.134 moles), with the previous $[5,12]$ and present concentration of $10^{-11} \mathrm{M}$ shown to be effective in human cells or with the doses reducing BP in the animal model [5].

The following arguments support the appropriateness of the available data to move to the phase 3 studies. Both OASIS-HT and PEARL-HT trials had baseline OSBP around $150 \mathrm{mmHg}$ and the degree of OSBP reduction produced by $50 \mu \mathrm{g}$ rostafuroxin ranged from 23.0 to 27.3 $\mathrm{mmHg}$ in either case in carriers of the "pre-specified" profile, which is of remarkable clinical significance for a drug acting on a causal mechanism of hypertension. This observation strengthens the choice of the dose of $50 \mu \mathrm{g}$ to move to phase 3 trial.
Both rat and human mutant $\alpha$-adducin and ouabain increase of Na-K pump units on the cell surface of renal tubular cells leading to an enhancement of renal sodium reabsorption [9-11, 16, 17]. Moreover, past [2, 4, 5, 12] and present findings show that rostafuroxin is able to selectively antagonize the effects of mutant $\alpha$-adducin and low concentrations of ouabain in vitro at concentration of $10^{-11} \mathrm{M}$, in rodent and human settings and in vivo, in a rodent model, without interacting with 35 others targets including hormones receptors or proteins involved in cardiovascular regulation up to concentration of $10^{-5} \mathrm{M}$.

Since rostafuroxin acts by selectively blocking a molecular mechanism common to both hypertension and organ damage [2, 4, 12, 30-32], for any given BP level, it may be endowed with an organ damage protection greater than the 20-30\% achievable with the available drugs.

The magnitude of the clinical and costs benefits may be inferred from the frequencies of the LSS AA genotype and P2a in Caucasians, about $10 \%$ and $20 \%$, respectively.

The major limitation of this study is the lack of BP response to rostafuroxin of Chinese patients being the magnitude of the losartan BP response similar to that of Caucasians. As discussed in the online Supplementary Data, in all the Randomized Controlled Trials (RCTs), the placebo treatment is associated with a significant $\mathrm{BP}$ fall ranging from 6 to $9 \mathrm{mmHg}$ [33]. The present findings suggested that in the Chinese P2 carriers the potential placebo effect associated to the rostafuroxin capsules administration was inhibited. Similarly, in the OASIS-HT study in Caucasian [13], placebo capsules significantly reduce BP in the general population of hypertensives, but they have no effect in carriers of the P2. The most logical explanation for this intriguing observation is to admit a faster metabolism or lower bioavailability of rostafuroxin in Chinese patients that were partially overridden by the dose of $500 \mu \mathrm{g}$.

Although the present results on Caucasian patients were obtained in a rather small sample size, they however showed a large effect size and replicates the results of 
OASIS-HT [13]. Moreover, according to Goodman [34] and Munafò [35], the degree of consistency of the present results with the previous ones discussed above, obtained both in humans and animals in different experimental and clinical settings, may strengthen the credibility of the present data.

If the present findings that are consistent with those of other two previous trials involving overall $658 \mathrm{Cau}-$ casian hypertensive patients were confirmed by the next phase 3 trial, they may contribute to the development of precision medicine in Caucasian patients with primary hypertension [36].

Acknowledgements We acknowledge the commitment of the PI of the various clinical centers enrolling patients. Italy: Francesco Perticone, Catanzaro; Flavio Scanferla, Mestre; Ferruccio Galletti, Napoli; Francesco Fallo, Padova; Angelo Rigotti, Rimini; Paolo Mulatero, Torino; Leonardo Sechi, Udine; Francesco Cocco, Manduria; Goffredo del Rosso, Teramo. Taiwan: Zhih-Cherng Chen, Tainan City; TsungHsien Lin, Kaohsiung City; Kuan-Cheng Chang, Taichung City; IChang Hsieh, Taoyuan City; Wei-Hsian Yin, Taipei City; Ming-En Liu, Hsinchu City; Kwo-Chang Ueng, Taichung City; Hung-I Yeh, Taipei City; Ting-Hsing Chao, Tainan City; Chih-Cheng Wu, Hsinchu City; Yen-Wen Wu, Taipei City; Wen-Chin Ko, Taipei City; ChangMin Chung, Chiayi.

Funding This study, funded by Cvie Therapeutics first and then by Windtree Therapeutics, is the last of a long series aimed at detecting a target "causal" molecular mechanism(s) of hypertension amenable to be "corrected" by a small molecule. To this end, it has been indispensable to set up parallel studies at the University Hospital VitaSalute San Raffaele, Milano and at pharmaceutical companies that changed over time (Prassis RostaQuo Sigma Tau, Settimo Milanese (MI); Cvie Therapeutics, Taipei; Windtree Therapeutics, Warrington, USA). Statistical analysis has been carried out first by the CRO statistician, then revised and supplemented by SC as statistician not involved in the previous activities.

\section{Compliance with ethical standards}

Conflict of interest GB, as it may be seen from the introduction and discussion, this study is the last of a long series aimed at detecting a target "causal" molecular mechanism(s) of hypertension amenable to be "corrected" by a small molecule in patients. To this end, GB was employee of the Università Vita Salute San Raffaele, Milano, Italy and consultant of pharmaceutical companies, that changed over time (Prassis RostaQuo Sigma Tau, Settimo M.se-Pomezia Italy; CVie Therapeutics, Taipei; Windtree Therapeutics, Warrington, USA), receiving a salary from the former and a consultancy fee from the latter, this being well known to both parties. In 2016, as Emeritus Professor, to comply with some local regulations, GB changed his position from consultant to employee at CVie Therapeutics first and then at Windtree; at present, he is consultant at Windtree. PF was consultant of CVie Therapeutics, Taipei, Taiwan first, and then of Windtree Therapeutics, Warrington, PA, USA. MF was an employee of Windtree Therapeutics, Warrington, PA, USA. SC received personal fees from Windtree Therapeutics, Warrington, USA. LFL was employee of CVie Therapeutics Taipei Taiwan. XL was CEO of CVie therapeutics Taipei Taiwan. CVie Therapeutics and Windree Therapeutics paid research grants for this study to the Institutions of GAS, NG, RB, CEC, TDW, KLW, PM, LC, DC, JAS, and CL have no conflicts of interest to declare.
Ethical statement All studies were approved by the local ethics committees and all participants provided written informed consent.

Publisher's note Springer Nature remains neutral with regard to jurisdictional claims in published maps and institutional affiliations.

Open Access This article is licensed under a Creative Commons Attribution 4.0 International License, which permits use, sharing, adaptation, distribution and reproduction in any medium or format, as long as you give appropriate credit to the original author(s) and the source, provide a link to the Creative Commons license, and indicate if changes were made. The images or other third party material in this article are included in the article's Creative Commons license, unless indicated otherwise in a credit line to the material. If material is not included in the article's Creative Commons license and your intended use is not permitted by statutory regulation or exceeds the permitted use, you will need to obtain permission directly from the copyright holder. To view a copy of this license, visit http://creativecommons. org/licenses/by/4.0/.

\section{References}

1. Manunta P, Rogowski AC, Hamilton BP, Hamlyn JM. Ouabaininduced hypertension in the rat: relationships among plasma and tissue ouabain and blood pressure. J Hypertens. 1994;12:549-60.

2. Ferrandi M, Molinari I, Barassi P, Minotti E, Bianchi G, Ferrari P. Organ hypertrophic signaling within caveolae membrane subdomains triggered by ouabain and antagonized by PST 2238. J Biol Chem. 2004;279:33306-14.

3. Ferrandi M, Manunta P, Balzan S, Hamlyn JM, Bianchi G, Ferrari P. Ouabain-like factor quantification in mammalian tissues and plasma: comparison of two independent assays. Hypertension. 1997;30:886-96.

4. Ferrari P, Ferrandi M, Valentini G, Bianchi G. Rostafuroxin: an ouabain antagonist that corrects renal and vascular $\mathrm{Na}+-\mathrm{K}+-$ ATPase alterations in ouabain and adducin-dependent hypertension. A J P Regul Integr Comp Physiol. 2006;290:R529-35.

5. Ferrari P. Rostafuroxin: an ouabain-inhibitor counteracting specific forms of hypertension. Biochim Biophys Acta (BBA)Molecular Basis of Disease. 2010;1802:1254-8.

6. Bianchi G, Tripodi G, Casari G, Salardi S, Barber BR, Garcia R, et al. Two point mutations within the adducin genes are involved in blood pressure variation. Proc Natl Acad Sci U.S.A. 1994;91:3999-4003.

7. Tripodi G, Valtorta F, Torielli L, Chieregatti E, Salardi S, Trusolino L, et al. Hypertension-associated point mutations in the adducin alpha and beta subunits affect actin cytoskeleton and ion transport. J Clin Invest. 1996;97:2815-22.

8. Cusi D, Barlassina C, Azzani T, Casari G, Citterio L, Devoto M, et al. Polymorphisms of alpha-adducin and salt sensitivity in patients with essential hypertension. Lancet. 1997;349:1353-57.

9. Bianchi G. Genetic variations of tubular sodium reabsorption leading to 'primary' hypertension: from gene polymorphism to clinical symptoms. Am J Physiol Regul Integr Comp Physiol. 2005;289:R1536-49.

10. Manunta P, Maillard M, Tantardini C, Simonini M, Lanzani C, Citterio L, et al. Relationships among endogenous ouabain, alphaadducin polymorphisms and renal sodium handling in primary hypertension. J Hypertens. 2008;26:914-20.

11. Citterio L, Lanzani C, Manunta P, Bianchi G. Genetics of primary hypertension: the clinical impact of adducin polymorphisms. Biochim Biophys Acta (BBA)—Molecular Basis of Disease. 2010;1802:1285-98.

12. Ferrandi M, Molinari I, Torielli L, Padoani G, Salardi S, Rastaldi $\mathrm{MP}$, et al. Adducin- and ouabain-related gene variants predict the 
antihypertensive activity of rostafuroxin, part 1: experimental studies. Sci Transl Med. 2010;2:59ra86-6.

13. Lanzani C, Citterio L, Glorioso N, Manunta P, Tripodi G, Salvi E, et al. Adducin- and ouabain-related gene variants predict the antihypertensive activity of rostafuroxin, part 2: clinical studies. Sci Transl Med. 2010;2:59ra87-7.

14. Frye SV. The art of the chemical probe. Nat Chem Biol. 2010; 6:159-61.

15. Manunta P, Ferrandi M, Cusi D, Staessen J, Bianchi G. Personalized therapy of hypertension: the past and the future. Curr Hypertens Rep. 2016;18:24-11.

16. Efendiev R, Krmar RT, Ogimoto G, Zwiller J, Tripodi G, Katz AI, et al. Hypertension- linked mutation in the adducin alpha-subunit leads to higher AP2-mu2 phosphorylation and impaired $\mathrm{Na}+, \mathrm{K}$ +-ATPase trafficking in response to GPCR signals and intracellular sodium. Circ Res. 2004;95:1100-8.

17. Torielli L, Tivodar S, Montella RC, Iacone R, Padoani G, Tarsini $\mathrm{P}$, et al. alpha-Adducin mutations increase $\mathrm{Na} / \mathrm{K}$ pump activity in renal cells by affecting constitutive endocytosis: implications for tubular Na reabsorption. Am J Physiol Renal Physiol. 2008;295: F478-87.

18. Blaustein MP. How does pressure overload cause cardiac hypertrophy and dysfunction? High-ouabain affinity cardiac $\mathrm{Na}+$ pumps are crucial. Am J Physiol Heart Circ Physiol. 2017;313: H919-30.

19. Fan X, Xie J, Tian J. Reducing cardiac fibrosis: Na/K-ATPase signaling complex as a novel target. Cardiovasc Pharm Open Access. 2017;6:pii:204.

20. Drummond CA, Fan X, Haller ST, Kennedy DJ, Liu J, Tian J. Na/ K-ATPase signaling mediates miR-29b-3p regulation and cardiac fibrosis formation in mice with chronic kidney disease. PLoS ONE. 2018;13:e0197688.

21. Cabrera CP, Ng FL, Nicholls HL, Gupta A, Barnes MR, Munroe $\mathrm{PB}$, et al. Over 1,000 genetic loci influencing blood pressure with multiple systems and tissues implicated. Hum Mol Genet. 2019;28:R151-61.

22. Wei WH, Hemani G, Haley CS. Detecting epistasis in human complex traits. Nat Rev Genet. 2014;15:722-33.

23. Tam V, Patel N, Turcotte M, Bosse Y, Paré G, Meyre D. Benefits and limitations of genome-wide association studies. Nat Rev Genet. 2019;20:467-84.
24. Munroe PB, Tinker A. Genome-wide association studies and contribution to cardiovascular physiology. Physiol Genomics. 2015;47:365-75.

25. Herrmann SM, Textor SC. Current concepts in the treatment of renovascular hypertension. Am J Hypertens. 2018;31:139-49.

26. Williams TA, Lenders JWM, Mulatero P, Burrello J, Rottenkolber $\mathrm{M}$, Adolf $\mathrm{C}$, et al. Outcomes after adrenalectomy for unilateral primary aldosteronism: an international consensus on outcome measures and analysis of remission rates in an international cohort. Lancet Diabetes Endocrinol. 2017;5:689-99.

27. Brown JJ, Davies DL, Ferriss JB, Fraser R, Haywood E, Lever $\mathrm{AF}$, et al. Comparison of surgery and prolonged spironolactone therapy in patients with hypertension, aldosterone excess, and low plasma renin. Br Med J. 1972;2:729-34.

28. Wald DS, Law M, Morris JK, Bestwick JP, Wald NJ. Combination therapy versus monotherapy in reducing blood pressure: metaanalysis on 11,000 participants from 42 trials. Am J Med. 2009;122: 290-300.

29. Takagi H, Mizuno Y, Niwa M, Goto SN, Umemoto T. A metaanalysis of randomized controlled trials of azilsartan therapy for blood pressure reduction. Hypertens Res. 2014;37:432-7.

30. Iatrino R, Lanzani C, Bignami E, Casamassima N, Citterio L, Meroni R, et al. Lanosterol synthase genetic variants, endogenous ouabain, and both acute and chronic kidney injury. Am J Kidney Dis. 2019;73:504-12.

31. Ferrandi M, Molinari I, Rastaldi MP, Ferrari P, Bianchi G, Manunta P. Rostafuroxin protects from podocyte injury and proteinuria induced by adducin genetic variants and ouabain. $\mathbf{J}$ Pharmacol Exp Ther. 2014;351:278-87.

32. Villa L, Buono R, Ferrandi M, Molinari I, Benigni F, Bettiga A, et al. Ouabain contributes to kidney damage in a rat model of renal ischemia-reperfusion injury. Int J Mol Sci. 2016;17:E1728.

33. Wilhelm M, Winkler A, Rief W, Doering BK. Effect of placebo groups on blood pressure in hypertension: a meta-analysis of betablocker trials. J Am Soc Hypertens. 2016;10:917-29.

34. Goodman SN. STATISTICS. Aligning statistical and scientific reasoning. Science. 2016;352:1180-1.

35. Munafò MR, Davey Smith G. Robust research needs many lines of evidence. Nature. 2018;553:399-401.

36. Kotchen TA, Cowley AW Jr, Liang M. Ushering hypertension into a new era of precision medicine. JAMA. 2016;315:343-4. 